\title{
Alteration of Time Intervals in Patients With Hypertrophic Cardiomyopathy During an Exercise Echocardiography
}

\author{
Angela Zagatina $^{\mathrm{a}, \mathrm{b}}$, Nadezhda Zhuravskaya ${ }^{\mathrm{a}}$, Tatyana V. Tyurina ${ }^{\mathrm{a}}$
}

\begin{abstract}
Background: Hypertrophic cardiomyopathy (HCM) is often associated with diastolic dysfunction. Theoretically, a more marked alteration of diastolic function is revealed during exercise.

Methods and Results: We studied 84 persons: 1) 25 patients with $\mathrm{HCM}$, 2) 25 patients with essential arterial hypertension (AH) and 3) 34 healthy controls. Each person performed a treadmill echocardiography. Before and after work, the following parameters were measured: the time interval between the QRS complex and the onset of mitral early diastolic filling velocity (TE), the interval between the QRS complex and the onset of peak early tissue mitral annular velocity (Te'), the isovolumetric relaxation time over the difference of TE and Te' ratio (IVRT/(TE-Te')), and changes of the time parameters during the stress test. In comparison with hypertensive and control groups, HCM patients at rest showed a significantly longer TE (448 \pm 55 vs. $423 \pm 33$ vs. $417 \pm 24 \mathrm{~ms}, \mathrm{P}$ $<0.04)$ and Te' $(446 \pm 48$ vs. $403 \pm 44$ vs. $416 \pm 38 \mathrm{~ms}, \mathrm{P}<0.003)$. After stress the HCM group had a longer Te' (355 \pm 59 vs. $299 \pm 40$ vs. $292 \pm 30 \mathrm{~ms}, \mathrm{P}<0.000004)$ and a higher IVRT/(TE-Te') ratio $(3.1 \pm 1.5$ vs. $0.9 \pm 2.4$ vs. $1.7 \pm 1.2, \mathrm{P}<0.002)$.
\end{abstract}

Conclusions: HCM patients show an alteration in the time parameters not only compared to healthy persons but to hypertensive patients as well.

Keywords: Hypertrophic cardiomyopathy; Diastolic function; Stress echocardiography

\footnotetext{
Manuscript accepted for publication April 19, 2011

${ }^{a}$ Leningrad Regional Cardiologic Dispensary, St. Petersburg, Russian Federation

${ }^{\mathrm{b}}$ Corresponding author: Angela Zagatina, Leningrad Regional Cardiologic Dispensary, 29-1-95, Vitebskiy Street, Saint-Petersburg, Russia, 196244. Email: zag_angel@yahoo.com
}

doi:10.4021/cr35w

\section{Introduction}

Hypertrophic cardiomyopathy is a genetic disease characterized by left ventricular hypertrophy and myocyte disarray [1-3]. This results in a delay in ventricular relaxation, an increase in left ventricle stiffness and a gradual elevation in filling pressure. Delayed relaxation is manifested by a prolonged isovolumetric relaxation time (IVRT) [4, 5]. With increasing left ventricular end-diastolic and filling pressures, IVRT becomes shorter, because there is an earlier equalization between left ventricle and left atrium pressures in diastole [4]. However, the time to reach peak early mitral annular velocity remains long. The time interval between the onset of early mitral diastolic filling velocity and the onset of early mitral annular velocity is strongly dependent on the time constant for left ventricle relaxation $[6,7]$.

Theoretically, patients with HCM, having diastolic dysfunction, should exhibit a difference in these time intervals in comparison with healthy persons. It should be more pronounced during exercise, because there would be a more marked alteration in diastolic function at those times.

The aim of the study was to investigate the alteration in the time intervals in HCM during exercise tests in comparison with healthy persons and to reveal the particulars of these alterations in comparison with patients having arterial hypertension.

\section{Methods}

\section{Subjects}

We studied 84 persons (3 groups): 1) 25 patients with HCM without any systolic obstructions in the left ventricular outflow tract at rest. The diagnostic criteria for HCM was a maximal left ventricular wall thickness greater than or equal to $15 \mathrm{~mm}$ in the absence of other cardiac or systemic disease [8]. 2) Twenty-five patients with arterial hypertension (comparable in their age and sex ratios). 3) Thirty-four healthy persons (comparable in their age and sex ratios).

Participants were excluded if they were not in sinus 


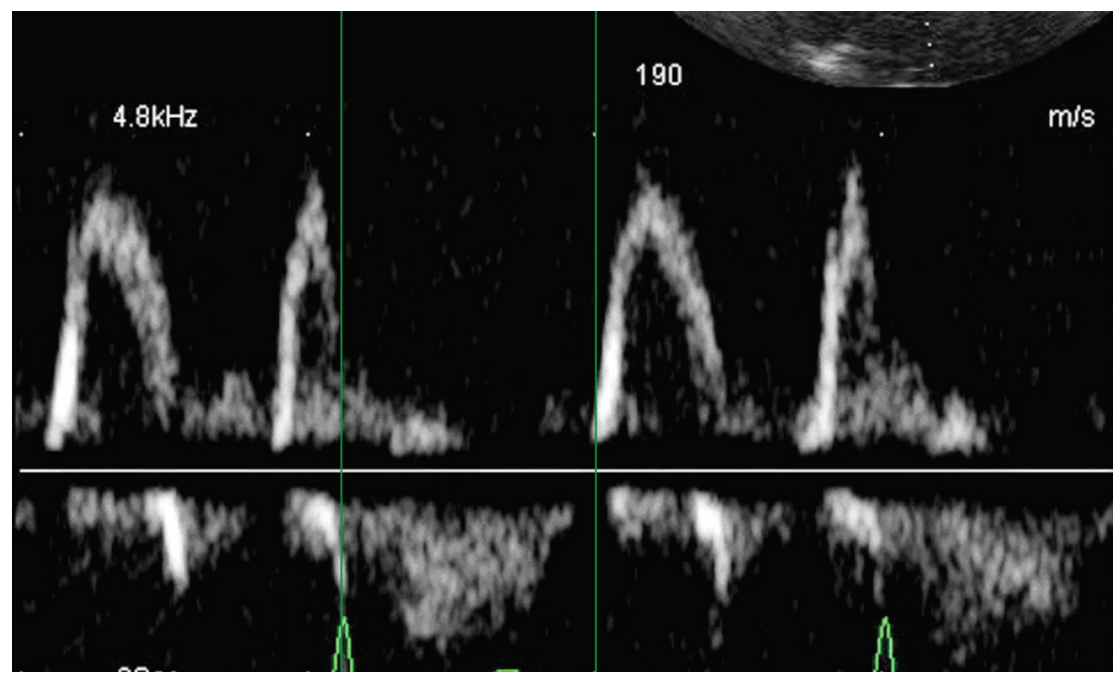

Figure 1. Time interval between the QRS complex and the onset of mitral early diastolic filling velocity - TE.

rhythm or if they had a history of coronary artery disease, regional wall abnormalities before or after the stress tests, valve disease, pericardial disease or cor pulmonale.

\section{Conventional echocardiography}

M-mode, 2D and Doppler standard echocardiographic examinations were performed in all subjects at rest, as proposed by respective guidelines $[9,10]$ using Sonoline G 60 S (Simens, Japan).

In the process the left atrial volume was measured using Simpson's rule. The left ventricular mass was estimated based on an area-length formula. For assessment and grading diastolic dysfunction we also measured and calculated trans- mitral flow parameters including the early (E) and late (A) diastolic filling velocities, the $\mathrm{E} / \mathrm{A}$ ratio, the $\mathrm{E}$ deceleration time (DT), and the A wave duration (Adur); isovolumetric relaxation time (IVRT); the ratio of the systolic (S) and diastolic (D) velocities (S/D), the late diastolic A wave (A') and duration of A' (A'dur) into the left atrium; the peak early diastolic lateral mitral annular velocities (e'); E/e' ratio; the difference in the pulmonary venous and the transmitral A wave durations (A'-Adur).

\section{Treadmill test}

Heart rate, blood pressure and a 12-lead ECG were obtained at baseline and at 3-min stages. Patients were encouraged to

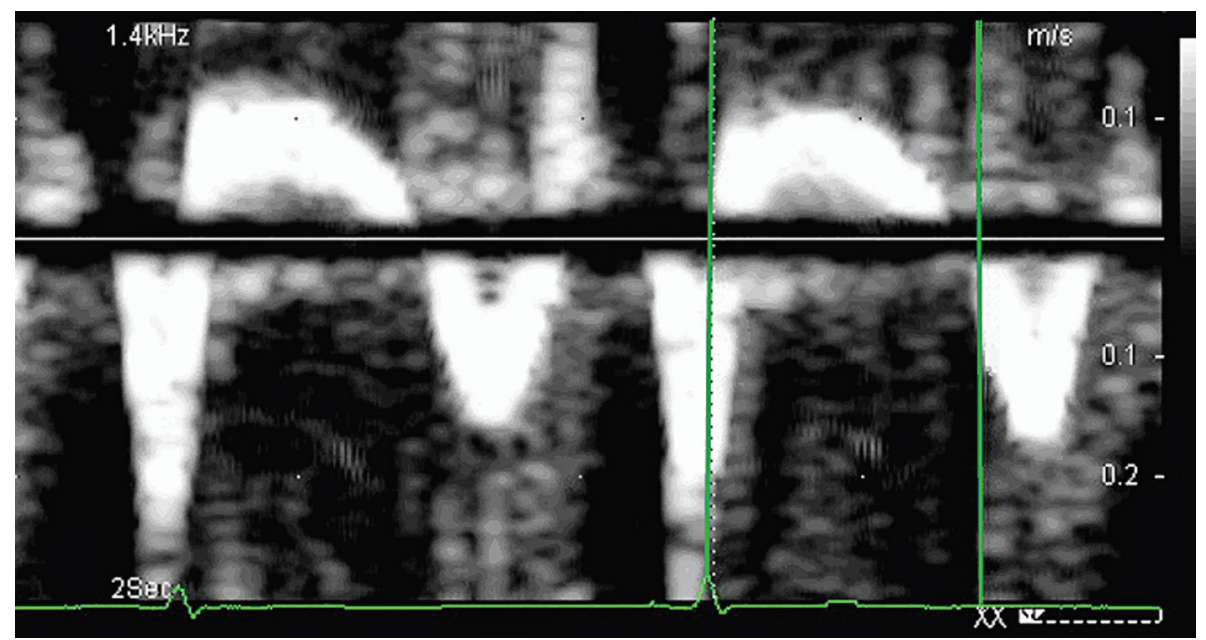

Figure 2. The interval between the QRS complex and the onset of peak early tissue mitral annular velocity - Te'. 
Table 1. General and Echocardiographic Parameters

\begin{tabular}{llllll}
\hline & HCM group & AH group & Normal controls & P-value 1 & P-value 2 \\
& N=25 & $\mathbf{N}=\mathbf{2 5}$ & $\mathbf{N}=\mathbf{3 4}$ & \\
\hline General data & & & & \\
Age (years) & $40 \pm 13$ & $46 \pm 7$ & $45 \pm 7$ & NS & NS \\
Gender (M/F) & $12 / 13$ & $17 / 8$ & $20 / 12$ & NS \\
BSA (m²) & $1.83 \pm 0.20$ & $2.00 \pm 0.21$ & $1.85 \pm 0.20$ & 0.006 & 0.003 \\
Echocardiographic data & & & & \\
IMM (g/m $\left.{ }^{2}\right)$ & $149 \pm 44$ & $86 \pm 24$ & $71 \pm 15$ & 0.0000001 & 0.008 \\
ILAVol (ml/m²) & $35 \pm 12$ & $29 \pm 8$ & $28 \pm 7$ & 0.04 & NS \\
IVRT (ms) & $101 \pm 24$ & $96 \pm 18$ & $80 \pm 16$ & NS & 0.0003 \\
E/A & $1.8 \pm 0.8$ & $1.2 \pm 0.3$ & $1.4 \pm 0.4$ & 0.0003 & 0.03 \\
DT (ms) & $198 \pm 53$ & $210 \pm 56$ & $182 \pm 36$ & NS & 0.005 \\
A duration (ms) & $113 \pm 24$ & $142 \pm 26$ & $133 \pm 30$ & 0.0003 & NS \\
PV S/D & $1.3 \pm 0.4$ & $1.3 \pm 0.3$ & $1.1 \pm 0.3$ & NS & 0.007 \\
PV A' (cm/s) & $0.32 \pm 0.07$ & $0.30 \pm 0.03$ & $0.30 \pm 0.05$ & NS & NS \\
A' duration (ms) & $115 \pm 34$ & $103 \pm 27$ & $93 \pm 17$ & 0.0008 & 0.05 \\
E/e' & $6.5 \pm 2.0$ & $4.7 \pm 1.5$ & $4.1 \pm 0.8$ & NS
\end{tabular}

HCM: hypertrophic cardiomyopathy; AH: arterial hypertension; BSA: body surface area; IMM: index myocardial mass; ILAVol: index of the left atrial volume; IVRT: isovolumetric relaxation time; E/A: ratio of the early filling (E) to the late diastolic filling (A) velocities; DT: deceleration time of the early diastolic velocity; PV S/D: ratio of the systolic (S) to early diastolic (D) velocities in the pulmonary vein; PV A': the late diastolic velocity in the pulmonary vein; E/e': ratio of the mitral to the annular early diastolic velocities; NS: non-significant difference.

P-value 1 was defined for comparison between $\mathrm{HCM}$ and $\mathrm{AH}$ groups.

$\mathrm{P}$-value 2 was defined for comparison between $\mathrm{AH}$ and control groups.

Table 2. Exercise End Points

\begin{tabular}{llll}
\hline & $\begin{array}{l}\text { HCM group } \\
\mathbf{N = \mathbf { 2 5 }}\end{array}$ & $\begin{array}{l}\text { AH group } \\
\mathbf{N = \mathbf { 2 5 }}\end{array}$ & $\begin{array}{l}\text { Normal controls } \\
\mathbf{N}=\mathbf{3 4}\end{array}$ \\
\hline Submaximal heart rate & $52 \%$ & $80 \%$ & $97 \%$ \\
Fatigue & $16 \%$ & $8 \%$ & $3 \%$ \\
Hypertensive response & $0 \%$ & $4 \%$ & $0 \%$ \\
Dyspnea & $12 \%$ & $6 \%$ & $0 \%$ \\
Drop in systolic blood pressure & $8 \%$ & $0 \%$ & $0 \%$ \\
Chest pain & $8 \%$ & $0 \%$ & $0 \%$ \\
\hline
\end{tabular}

HCM: hypertrophic cardiomyopathy; $\mathrm{AH}$ : arterial hypertension 
Table 3. Echocardiographic Parameters of Diastolic Function After the Stress

\begin{tabular}{lllll}
\hline & $\begin{array}{l}\text { HCM group } \\
\mathbf{N}=\mathbf{2 5}\end{array}$ & $\begin{array}{l}\text { AH group } \\
\mathbf{N}=\mathbf{2 5}\end{array}$ & $\begin{array}{l}\text { Normal controls } \\
\mathbf{N}=\mathbf{3 4}\end{array}$ & P-value \\
\hline IVRT (ms) & $80 \pm 20$ & $82 \pm 14$ & $66 \pm 12$ & NS \\
E/A & $1.7 \pm 0.6$ & $1.1 \pm 0.3$ & $1.3 \pm 0.3$ & 0.0003 \\
DT (ms) & $174 \pm 44$ & $184 \pm 54$ & $175 \pm 32$ & NS \\
A duration (ms) & $118 \pm 25$ & $127 \pm 29$ & $134 \pm 29$ & NS \\
PV S/D & $1.2 \pm 0.5$ & $1.3 \pm 0.3$ & $1.2 \pm 0.2$ & NS \\
PV A' (cm/s) & $0.34 \pm 0.08$ & $0.34 \pm 0.04$ & $0.34 \pm 0.06$ & NS \\
A' duration (ms) & $110 \pm 24$ & $100 \pm 15$ & $93 \pm 17$ & NS \\
E/e' & $5.9 \pm 2.0$ & $4.3 \pm 1.4$ & $3.9 \pm 0.9$ & 0.007 \\
\hline
\end{tabular}

HCM: hypertrophic cardiomyopathy; $\mathrm{AH}$ : arterial hypertension; IVRT: isovolumetric relaxation time; E/A: ratio of the early filling (E) to the late diastolic filling (A) velocities; DT: deceleration time of the early diastolic velocity; PV S/D: ratio of the systolic (S) to early diastolic (D) velocities in the pulmonary vein; PV A': the late diastolic velocity in the pulmonary vein; E/e': ratio of the mitral to the annular early diastolic velocities; NS: non-significant difference.

$\mathrm{P}$-value was defined for comparison between HCM and $\mathrm{AH}$ groups.

perform a treadmill exercise test (Bruce protocol, modified Bruce) until exhaustion or until they reached an endpoint. Endpoints were standard for exercise tests [11].

\section{Stress echocardiography}

Before and after maximal tolerated workloads the following parameters were measured and calculated: the time interval between the QRS complex and the onset of mitral early diastolic filling velocity (TE) (Fig. 1), the interval between the QRS complex and the onset of peak early tissue mitral annular velocity (Te') (Fig. 2), the isovolumetric relaxation time over the difference between the TE and Te' (IVRT/(TE-Te')), the change of post-stress and rest TE $(\triangle \mathrm{TE})$, and the change of post-stress and rest Te' $\left(\Delta \mathrm{Te} \mathrm{e}^{\prime}\right)$. All the post-stress images were acquired till 5 th minutes after stress. Image acquisition was performed on-line and stored for off-line analysis. All patients performed without beta-blockers.

\section{Statistical analysis}

Continuous variables were described by means and standard deviations (STATISTICA version 6). For multiple comparisons ANOVA was performed. Mann-Whitney U Test was used for comparison of ratios. Pearson Chi-square was used for crosstabulation tables. Wilcoxon matched pairs test was used for comparing changes in the time ratios during exercise. Significance was determined as $\mathrm{P}<0.05$.

\section{Results}

\section{General data and rest echocardiography}

Eleven of the HCM patients (44\%) had a history of dyspnea. General and echocardiographic parameters for diastolic function are summarized in Table 1. All the patients had normal systolic function before and after the stress tests. Despite the significant abnormality of the left ventricular mass index, and left atrial volume index, only four patients (16\%) with HCM at rest had a classified type of diastolic dysfunction according to "Recommendations for the Evaluation of Left Ventricular Diastolic Function" [12]. The majority of HCM patients (75 - 85\%) had E/A, DT, A'-Adur and S/D values within normal range. At the same time IVRT was longer in $56 \%$ of HCM patients. The ratio of the mitral early diastolic to the annular early diastolic velocities (E/e') was less than 8 in 22 persons with $\mathrm{HCM}(88 \%)$, and from 8 to 15 in 3 patients. One of the latter had no other signs of abnormal filling and diastolic dysfunction.

The patients in the AH group had histories of high blood pressure for $6.7 \pm 5.7$ years (more than 5 years in 16 patients, 64\%). Like the HCM groups at rest, the majority of $\mathrm{AH}$ patients (18 subjects, $72 \%$ ) had no signs of diastolic dysfunction. Five of them $(20 \%)$ had impaired relaxation patterns and two patients $(8 \%)$ had pseudonormal types of diastolic dysfunction. The AH group was comparable with the HCM group in their age and sex ratios so these patients 
Table 4. The Relative Time Parameters

\begin{tabular}{|c|c|c|c|c|}
\hline & $\begin{array}{l}\text { HCM group } \\
N=25\end{array}$ & $\begin{array}{l}\text { AH group } \\
\mathbf{N}=\mathbf{2 5}\end{array}$ & $\begin{array}{l}\text { Normal controls } \\
\mathbf{N}=\mathbf{3 4}\end{array}$ & P-value \\
\hline TE/R-R rest & $0.49 \pm 0.07$ & $0.51 \pm 0.07$ & $0.50 \pm 0.05$ & NS \\
\hline Te'/R-R rest & $0.48 \pm 0.07$ & $0.48 \pm 0.06$ & $0.50 \pm 0.05$ & NS \\
\hline TE/R-R post stress & $0.52 \pm 0.05$ & $0.53 \pm 0.06$ & $0.53 \pm 0.04$ & NS \\
\hline Te'/R-R post stress & $0.54 \pm 0.07$ & $0.49 \pm 0.06$ & $0.49 \pm 0.06$ & 0.03 \\
\hline
\end{tabular}

HCM: hypertrophic cardiomyopathy; $\mathrm{AH}$ : arterial hypertension; TE/R-R: ratio, the time interval between the QRS complex and the onset of mitral early diastolic filling velocity/the time of R-R interval; Te'/R-R: ratio, the interval between the QRS complex and the onset of peak early tissue mitral annular velocity/the time of R-R interval; NS: non-significant difference.

were younger-aged with a lesser degree of arterial hypertension in comparison with the general population. In spite of that, these patients had significant differences in their BSA, IMM, TD, IVRT, S/D and E/e' than controls (Table 1).

\section{Medical treatment}

Eleven of the HCM patients (44\%) were being treated with beta-blockers. Sixteen patients in the AH group (64\%) were being given beta-blockers, nine subjects (36\%) - ACE inhibitors, and 2 patients were additionally treated with thiazide diuretics.

All the medication had been stopped prior to the test.

\section{Treadmill test}

The reasons for terminating exercise are summarized in Table 2 .

The maximum heart rate of HCM patients was $147 \pm 21$ $\mathrm{bpm}$, the maximum heart rate of patients with arterial hypertension was $153 \pm 13 \mathrm{bpm}(\mathrm{P}=0.18)$. There were significantly higher heart rates in control persons $(158 \pm 8 \mathrm{bpm}$, $\mathrm{P}<0.009$ ). Maximum workload achieved on the treadmill was $10.5 \pm 4.2$ MET for HCM patients, $11.3 \pm 3.1$ MET for patients with arterial hypertension $(\mathrm{P}=0.42)$ and $12.0 \pm 4.0$ MET for controls $(\mathrm{P}=0.18)$.

After exercise, seven of the HCM persons (28\%) had a significant obstruction in the left ventricular outflow tract.

The echocardiographic parameters of diastolic function immediately after exercise are summarized in Table 3 .

\section{Time parameters}

In comparison with hypertensive and control groups, HCM patients at rest showed a significantly longer TE $(448 \pm 55$ vs. $423 \pm 33$ vs. $417 \pm 24 \mathrm{~ms}, \mathrm{P}<0.04)$ and Te' $(446 \pm 48$ vs. $403 \pm 44$ vs. $416 \pm 38 \mathrm{~ms}, \mathrm{P}<0.003)$. After stress the HCM group had a longer Te' $(355 \pm 59$ vs. $299 \pm 40$ vs. $292 \pm 30$ $\mathrm{ms}, \mathrm{P}<0.000004)$ and a higher IVRT/(TE-Te') ratio (3.1 \pm 1.5 vs. $0.9 \pm 2.4$ vs. $1.7 \pm 1.2, \mathrm{P}<0.002)$. The time parameters for patients with hypertension did not significantly differ from controls before and after the exercise test.

There was not a significant difference between the peak heart rates of HCM and AH groups. However, all the parameters were obtained after stress during 5 minutes when the velocity of heart rate recovery was different in different patients. In addition the control group had a higher peak heart rate. Taking into account that the time parameters are significantly dependent on heart rate, we assessed the relation of these parameters to R-R duration in the acquired cycle.

The obtained data showed time TE (until mitral valve opening) to the overall time of R-R interval, and time Te' (until the onset of diastolic wall motion relaxation) to the overall time of R-R interval (Table 4).
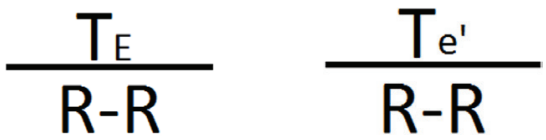

The $\mathrm{AH}$ and control groups had the same relative time until the onset of diastolic wall motion relaxation before and after the stress. That is Te'/R-R was the same before and immediately after the exercise in these groups. Whereas, this ratio $(\mathrm{Te} / \mathrm{R}-\mathrm{R})$ increased significantly after the exercise in HCM patients $(0.48 \pm 0.07$ vs. $0.54 \pm 0.07, \mathrm{P}<0.0004)$.

\section{Discussion}

Diastolic dysfunction is important to assess for many reasons. There are numerous studies that have established the prognostic significance of diastolic dysfunction signs [1316]. Echocardiography remains the key modality in its estimation but is not without limitation. It is especially inconclusive for particular groups of patients. Present methods are 
not effective enough in determining diastolic dysfunction and assessing left ventricular filling pressure in HCM. Previous studies have shown that in contrast to other diseases, the mitral variables in the ratio 'peak early filling to late diastolic filling velocities' (E/A ratio) and deceleration time (DT), have weak to no correlation with the grade of diastolic dysfunction and left ventricle filling pressures in patients with HCM [17]. There are also discrepancies in the data about the application of the well-known ratio 'peak mitral early diastolic filling velocity to early mitral annular velocity' (E/e' ratio) in the assessment of diastolic dysfunction and filling pressure [18-20].

In our study we selected the group of HCM patients which had no contraindications to exercise. Thereby all the subjects with an obstruction in the left ventricle outflow tract at rest were excluded. As a result our group came out as a sample of younger-aged patients with less manifestations of heart failure in comparison with the parent population. At rest this group had safe systolic and diastolic function according to conventional assessment. However, half of them had a history of dyspnea, a third of them presented a significant rise in the left ventricular outflow pressure gradient after stress, and an abnormal blood pressure response to exercise tests occurred in two patients. So abnormal tests were obtained in more than half of the cases. It is likely that these patients have some diastolic dysfunction especially during exertion, but present methods are not sensitive enough for detection in these cases.

In our present investigations we observed time parameters (TE, Te', IVRT/(TE-Te')) which were significantly different from controls and even from patients with arterial hypertension. Theoretically, these parameters can unmask impaired diastolic function in patients with $\mathrm{HCM}$ at rest and during exercise.

Relative time parameters TE/R-R and Te'/R-R likely reveal true changes in time parameters during exercise. Also an increase of Te'/R-R immediately after the stress indicates the delay of diastolic relaxation while decreasing IVRT or the absence of such significant changes of TE/R-R could be due to increasing pressure in the left atrium and a more rapid opening of the mitral valve. These changes were observed in HCM patients.

Previous investigations [18-20] have shown a bigger mean value of $\mathrm{E} / \mathrm{e}$ ' in patients with $\mathrm{HCM}$ than was in our study. That can be explained by the different samples of subjects. The above-mentioned studies were confined to patients who were selected for operations. As the result, these people presented significant left ventricle outflow tract obstructions at rest, III-IV NYHA class of heart failure and more pronounced diastolic dysfunction.

\section{Study limitations}

The patient selection was confined to those being in sinus rhythm, and HCM subjects being non-obstructive at rest.

We did not perform invasive measurements of the mean LV diastolic pressure for estimation of the mean left atrial pressure at rest and during exercise.

The number of patients in HCM group was limited for the purpose of dividing it into subgroups with and without abnormal test.

The group of AH was comparable with HCM group in their age and sex ratios so the result was also comprised of younger-aged patients with less degree of arterial hypertension.

The present data remains to be verified in future studies.

\section{References}

1. Bonne G, Carrier L, Richard P, Hainque B, Schwartz K. Familial hypertrophic cardiomyopathy: from mutations to functional defects. Circ Res 1998;83(6):580-593.

2. Marian AJ, Roberts R. Molecular genetics of hypertrophic cardiomyopathy. Annu Rev Med 1995;46:213-222.

3. Schwartz K, Carrier L, Guicheney P, Komajda M. Molecular basis of familial cardiomyopathies. Circulation 1995;91(2):532-540.

4. Alvares RF, Shaver JA, Gamble WH, Goodwin JF. Isovolumic relaxation period in hypertrophic cardiomyopathy. J Am Coll Cardiol 1984;3(1):71-81.

5. Slama M, Ahn J, Peltier M, Maizel J, Chemla D, Varagic $\mathrm{J}$, Susic D, et al. Validation of echocardiographic and Doppler indexes of left ventricular relaxation in adult hypertensive and normotensive rats. Am J Physiol Heart Circ Physiol 2005;289(3):H1131-1136.

6. Rivas-Gotz C, Khoury DS, Manolios M, Rao L, Kopelen HA, Nagueh SF. Time interval between onset of mitral inflow and onset of early diastolic velocity by tissue Doppler: a novel index of left ventricular relaxation: experimental studies and clinical application. J Am Coll Cardiol 2003;42(8):1463-1470.

7. Hasegawa H, Little WC, Ohno M, Brucks S, Morimoto A, Cheng HJ, Cheng CP. Diastolic mitral annular velocity during the development of heart failure. J Am Coll Cardiol 2003;41(9):1590-1597.

8. Maron BJ, McKenna WJ, Danielson GK, Kappenberger LJ, Kuhn HJ, Seidman CE, Shah PM, et al. American College of Cardiology/European Society of Cardiology clinical expert consensus document on hypertrophic cardiomyopathy. A report of the American College of Cardiology Foundation Task Force on Clinical Expert Consensus Documents and the European Society of Cardiology Committee for Practice Guidelines. J Am Coll Cardiol 2003;42(9):1687-1713.

9. Lang RM, Bierig M, Devereux RB, Flachskampf FA, Foster E, Pellikka PA, Picard MH, et al. Recommen- 
dations for chamber quantification: a report from the American Society of Echocardiography's Guidelines and Standards Committee and the Chamber Quantification Writing Group, developed in conjunction with the European Association of Echocardiography, a branch of the European Society of Cardiology. J Am Soc Echocardiogr 2005;18(12):1440-1463.

10. Quinones MA, Otto CM, Stoddard M, Waggoner A, Zoghbi WA. Recommendations for quantification of Doppler echocardiography: a report from the Doppler Quantification Task Force of the Nomenclature and Standards Committee of the American Society of Echocardiography. J Am Soc Echocardiogr 2002;15(2):167184.

11. Gibbons RJ, Balady GJ, Bricker JT, Chaitman BR, Fletcher GF, Froelicher VF, Mark DB, et al. ACC/AHA 2002 guideline update for exercise testing: summary article: a report of the American College of Cardiology/ American Heart Association Task Force on Practice Guidelines (Committee to Update the 1997 Exercise Testing Guidelines). Circulation 2002;106(14):18831892.

12. Nagueh SF, Appleton CP, Gillebert TC, Marino PN, Oh JK, Smiseth OA, Waggoner AD, et al. Recommendations for the evaluation of left ventricular diastolic function by echocardiography. J Am Soc Echocardiogr 2009;22(2):107-133.

13. Redfield MM, Jacobsen SJ, Burnett JC, Jr., Mahoney DW, Bailey KR, Rodeheffer RJ. Burden of systolic and diastolic ventricular dysfunction in the community: appreciating the scope of the heart failure epidemic. JAMA 2003;289(2):194-202.

14. Wang M, Yip GW, Wang AY, Zhang Y, Ho PY, Tse MK, $\mathrm{Yu}$ CM, et al. Tissue Doppler imaging provides incre- mental prognostic value in patients with systemic hypertension and left ventricular hypertrophy. J Hypertens 2005;23(1):183-191.

15. Wang M, Yip GW, Wang AY, Zhang Y, Ho PY, Tse MK, Lam PK, et al. Peak early diastolic mitral annulus velocity by tissue Doppler imaging adds independent and incremental prognostic value. J Am Coll Cardiol 2003;41(5):820-826.

16. Okura H, Takada Y, Kubo T, Iwata K, Mizoguchi S, Taguchi H, Toda I, et al. Tissue Doppler-derived index of left ventricular filling pressure, E/E', predicts survival of patients with non-valvular atrial fibrillation. Heart 2006;92(9):1248-1252.

17. Nagueh SF, Lakkis NM, Middleton KJ, Spencer WH, 3rd, Zoghbi WA, Quinones MA. Doppler estimation of left ventricular filling pressures in patients with hypertrophic cardiomyopathy. Circulation 1999;99(2):254261.

18. Geske JB, Sorajja P, Nishimura RA, Ommen SR. Evaluation of left ventricular filling pressures by Doppler echocardiography in patients with hypertrophic cardiomyopathy: correlation with direct left atrial pressure measurement at cardiac catheterization. Circulation 2007;116(23):2702-2708.

19. Nishimura RA, Appleton CP, Redfield MM, Ilstrup DM, Holmes DR, Jr., Tajik AJ. Noninvasive doppler echocardiographic evaluation of left ventricular filling pressures in patients with cardiomyopathies: a simultaneous Doppler echocardiographic and cardiac catheterization study. J Am Coll Cardiol 1996;28(5):1226-1233.

20. Eriksson MJ, Jacobsson L, Tronnberg S, Lundh A, Rickenlund A, Caidahl K Svane B, Gadler F. Doppler-Echocardiographic estimation vs. invasive measurement. Eur J Echocardiogr 2009;10:S.143. 